\title{
会吉
}

\section{John Stuart Mill: la etapa de madurez de la escuela clásica}

\author{
Carmen Pelet Redón \\ DEPARTAMENTO DE ESTRUCTURA E HISTORIA ECONÓMICA \\ Y ECONOMÍA PÚBLICA \\ UNIVERSIDAD DE ZARAGOZA
}

John Stuart Mill es considerado como uno de los principales representantes de la Escuela Clásica de pensamiento económico. Su obra los "Principios", publicada en 1848, fue referencia obligada durante más de cuarenta años en los ámbitos intelectuales y académicos ingleses de su época; en ella realiza un considerable esfuerzo por acercar las ideas económicas del plano teórico al aplicado y sin duda lo consiguió. Su biografía personal e intelectual demuestran una clara vocación de reformador.

En España, la difusión de su obra no sería un hecho hasta la Restauración (1874), quizás por la fuerte hegemonía, durante el periodo, de la Escuela Economista francesa que condicionaron, a su vez, la tardía recepción de las ideas marginalistas, retrasando la incorporación de la nueva economía a la enseñanza y la investigación hasta la puesta en funcionamiento de la Facultad de Ciencias Políticas y Económicas de Madrid en 1943.

Palabras claves. John Stuart Mill. Economía Política. La Escuela Clásica en España. 


\section{John Stuart Mill: la etapa de madurez}

de la escuela clásica

Carmen Pelet Redón

\section{El escenario de la Economía Clásica}

\subsection{La discusión del método}

Uno de los rasgos característicos del periodo que comprende el desarrollo de la Escuela Clásica es el deseo de los economistas de teorizar sus propias aspiraciones y procedimientos. La aparición de este interés, casi ausente hasta entonces, indica que la economía había logrado cierta madurez. Sabemos que los economistas habían sentido ya antes de A. Smith la necesidad de definir su campo pero fue durante este periodo, llamado de la Escuela Clásica, cuando su consciencia de pertenecer a un campo específico de la ciencia se ve fortalecido de forma que todos los autores intentarán definirlo. La ciencia que se trataba de definir era normalmente llamada Economía política. Todas las definiciones formuladas en este periodo señalan la autonomía de la economía respecto de las demás ciencias sociales o morales perfectamente compatible, por otra parte, con las estrechas vinculaciones entre ellas. La mayoría de las definiciones subrayan el carácter analítico de la economía. La definición que ofrece J. S. Mill de Economía política, como la de la mayoría de los autores clásicos, (Say, Señor, McCulloch, etc.) no es muy especifica.J.S. Mill se ocupa en los «Principios» de: «la naturaleza de la riqueza y las leyes de su producción y distribución, incluyendo, directa o en forma remota, la actualización de todas las causas por las que la situación de la humanidad, o de cualquier sociedad de seres humanos, prospera o decae respecto de ese 
objetivo universal de los deseos humanos» (Mill, J.S. 1978, p.29).

Aunque, la discusión sobre el método no había empezado todavía en este periodo, los clásicos teorizaban para aclarar puntos que acarreaban controversias lógicas, y reunían hechos cuando les parecía útil o necesario hacerlo. La mayoría de los economistas del periodo y entre ellos J.S.Mill estudió la analogía entre la economía y las ciencias físicas. Mill (1873, p.163) consideró «verdaderos modelos para el análisis en la Teoría Económica el proceso deductivo de las ramas deductivas de la filosofía natural ». No debemos olvidar que Mill está pensando en el aparato teórico de la economía al referirse a los métodos de la Economía política. La mayoría de los autores del periodo que prestaron atención a las cuestiones metodológicas señalaron la distinción entre la «ciencia» económica y el «arte» de la política económica.Mill y sus contemporáneos pensaban que las cuestiones de política económica implican tantos elementos no económicos que no se debían tratar sobre la base de consideraciones exclusivamente económicas; sin embargo sería un error pensar que estos autores consideraron los juicios de valor.Los economistas de ese periodo consideraban sus recomendaciones de política económica como resultados científicos resultantes de un análisis científico, aunque no puramente económico.Los «clásicos», J.S.Mill incluido, realizaron importantes progresos en el logro de un aparato analítico que unificara todos los problemas puramente económicos.

1.2 Los criterios clásicos de política económica: el papel del Estado

El estereotipo de la Escuela Clásica como defensora a ultranza del laissez-faire viene matizada al examinar los escritos clásicos acerca del papel del Estado en la economía. Así lo expresa J.S.Mill (1848, p.800):

«Las funciones admisibles del Estado abarcan un campo cuyas fronteras superan con mucho las de cualquier definición restrictiva y (...) es casi imposible señalar una sola base para justificarlas a todas, excepto la muy amplia de la utilidad general.»

Si bien es cierto que los economistas clásicos, algo especialmente cierto en J.S.Mill, temen por el acrecentamiento del poder del Estado que hacía peligrar la libertad individual. 
El papel fundamental que los economistas clásicos asignan al Estado, desde Smith en adelante, comprende la defensa, la justicia, el suministro de infraestructura básica y otros servicios como la acuñación de moneda y el correo, el establecimiento de impuestos etc. Hubo también otros campos donde los economistas clásicos juzgaron necesaria la intervención pública:el trabajo infantil, la educación y los pobres.

No obstante, hay a juicio de los clásicos una serie de áreas donde la intervención del Estado es claramente reprobable, en especial, la fijación de precios y la reglamentación de los procesos industriales, puesto que para los clásicos, la interferencia en este campo podía frenar la innovación.

Por último, está la cuestión del socialismo y el orden social. Los clásicos aceptaron ampliamente el régimen de propiedad privada. La propiedad y la seguridad eran componentes esenciales del incentivo para el crecimiento económico. Sólo Bentham y J. S. Mill apoyaron planes para la reforma de la propiedad y la herencia. Sólo J. S. Mill mostró autentica simpatía hacia el socialismo. En un principio, su posición con respecto al socialismo fue de duda sobre la innovación, los incentivos, la distribución y la evolución probable de la población bajo un sistema socialista. Posteriormente, influido por las ideas de su esposa Harriet Taylor, Mill modificó su posición reflejando una actitud más favorable, sin embargo, su postura volvió a sus orígenes, tras el fallecimiento de su esposa. A lo largo de todas estas vicisitudes, algo destaca y es que el socialismo que Mill tenía en su pensamiento era el de Fourier, Saint Simón, y Owen, un socialismo descentralizado en el que compiten las cooperativas de producción.El socialismo de corte centralista no le atrajo nunca.

\section{La figura de John Stuart Mill}

Para comprender el objeto que J. S. Mill perseguía con sus estudios económicos, cual era el de transformar la Economía política, se hace necesario conocer su biografía intelectual resaltando las influencias de sus maestros, así como sus relaciones con los filósofos y otras corrientes de pensamiento presentes en su tiempo. 
No es excesiva la importancia que se le atribuye a la influencia que sobre J.S. Mill tuvo su padre James Mill, la rígida educación que de él recibió le marcó indeleblemente, tanto en los primeros años de infancia y juventud, como en momentos posteriores de su vida; y aunque intentó acercamientos a otras posiciones ideológicas o planteamientos intelectuales nunca logró liberarse por completo de aquella influencia paterna.

Otra influencia notable fue los escritos de Bentham, gran amigo de su padre y con quien llegó a convivir algún tiempo, Mill acogió desde un principio con gran entusiasmo los planteamientos del Utilitarismo.

Un hecho notable en la biografía personal e intelectual de J. S. Mill fue su relación con la sufragista Harriet Taylor, quien llegará a convertirse en su esposa, ella despertó en Mill su interés por la economía aplicada, por su lado práctico, el marco institucional y las consecuencias políticas de la economía. Harriet Taylor tuvo una gran influencia en los escritos de Mill y en su propia evolución espiritual.

John Stuart Mill fue un gran intelectual y un rápido repaso a su intensa biografía lo pone, sin duda, de manifiesto. Nació en Londres en 1806 en una familia numerosa y de escasos ingresos, era el hijo mayor de James Mill, hijo a su vez de un modesto granjero y pequeño comerciante. Su padre, James Mill era un presbiteriano escocés que fue ordenado Predicador aunque nunca ejerció; educó a J. S. Mill sin ningún tipo de creencia religiosa. El carácter estoico del padre, imbuido por las ideas de la llustración y por el carácter de los filósofos griegos, modeló el carácter del hijo transmitièndole sus fuertes convicciones morales; valores como la justicia, templanza, sinceridad, perseverancia, trabajo, la vida de esfuerzo, disposición para afrontar el dolor, respeto por el bien común, estimación de las personas de acuerdo con sus méritos, estimación de las cosas por su utilidad intrínseca. Puede decirse que una de las principales actividades en su vida fue la instrucción de sus hijos, principalmente de J.S.Mill. James Mill, llegó a convertirse en economista tras su educación en la Universidad de Edimburgo, fue autor de obras como los «Elementos de Economía Política» y escribió, así mismo, "La Historia de la India» en la que sentó los fundamentos para la administración de la India, ámbito del que era conocedor por su labor como funcionario de la India House, encar- 
gado de redactar los despachos que se enviaban a la India. J. S. Mill también trabajaría en la Compañía de la India, durante treinticinco años.

Durante sus primeros años, J. S. Mill y su familia vivían en una pequeña aldea, sus primeros recuerdos están asociados con ese entorno y con el hábito de dar largos paseos con su padre durante los cuales aprovechaba para transmitirle todo lo que había leído, esta era la forma en que se desarrolló la educación de Mill en su infancia. Con tres años leía griego, su primer libro en griego fueron las Fábulas de Esopo posteriormente leería a Herodoto, Sócrates y Platón y las tardes las dedicaba a aprender Aritmética.La mayoría de los libros que leía eran libros de Historia, de autores como Robertson, Hume, Gibbon, Watsonsobre, La Historia de Roma de Looke, Libros de Historia Eclesiástica, y biografías de hombres luchadores con gran energía, apenas leía libros infantiles, solo como regalo contó con las obras de Robinson Crusoe, su favorita, «Las mil y una noches», Cuentos Árabes y «El Quijote».

A los ocho años de edad aprendía Latín y se lo enseñaba a sus hermanos pequeños; entre los ocho y los trece años, leyó obras de poetas griegos como la «lliada», «Eneida», "La retórica» de Aristóteles compartiéndolo con el estudio del álgebra, el cálculo diferencial, aunque de forma superficial, nunca fue un buen matemático, tratados de Física y Química, Tratados de lógica y de lógica escolástica; a Shakespeare y los poemas de Campbell.

En 1819 comienza un curso de Economía Política con su padre y con Ricardo gran amigo de su padre, por aquel entonces frecuentaban también la compañía de Bentham todos ellos ejercieron una gran influencia en la educación de J.S.Mill.En 1820 pasa un año en Francia en casa de Say amigo de su padre, de Saint Simón y de todos los líderes del partido liberal republicano lo que despertó el interés de Mill por el liberalismo contintental. Durante 1822-23 J.S.Mill empezó a escribir en los periódicos bajo seudónimo plasmando sus opiniones en materia de política y de religión.En 1824-25 Bentham le encarga preparar el manuscrito para publicar su obra «La teoría de la Evidencia Judicial». Desde 1821 año en que lee por primera vez a Bentham su meta será convertirse en reformador del mundo. En esta época aprende Alemán y crea una sociedad que llamó 
Sociedad Utilitarista formada por un grupo de jóvenes a la que acude a reuniones periódicas para discutir temas de su interés que deseaban conocer y dominar, comenzaron con el libro de su padre los «Elementos», y continuaron con los «Principios de Economía Política» de Ricardo, de estas conversaciones saldría posteriormente su Teoría sobre los Valores Internacionales y su versión modificada de la Teoría del Beneficio de Ricardo que aparece en sus «Ensayos sobre el Beneficio y el Interés». Durante estos años comenzó a hablar en público participando en debates públicos celebrados por distintas sociedades, entre ellas la Sociedad Cooperativa seguidores de Owen. Hasta 1830 es un periodo en el que no escribe y se dedica a reflexionar. Durante estos años de transición sintió la influencia de los Santsimonianos y de Comte, leyó sus críticas al liberalismo lo que le llevó a abrir los ojos al limitado valor de la vieja Economía política basada en las ideas de la propiedad privada y la herencia, así como de la libertad de producción y cambio como pilares de la mejora social, su transformación personal le llevó adoptar nuevas ideas políticas próximas a la democracia y al socialismo fundamentadas en una defensa de la igualdad entre los hombres y en la importancia de la educación del pueblo para alcanzar esos ideales. Sin duda, no podemos olvidar la influencia que tuvo en toda esta evolución el encuentro que tuvo lugar por aquel entonces con Harriet Taylor con quien estuvo casado desde 1851 hasta 1858 año en que ella murió.

Durante 1830-31 escribió los cinco ensayos publicados en 1844 como «Ensayos sobre algunas discutidas cuestiones de Economía Política», entre 1834 y 1840 fue director de la revista London Review, en 1837 reanuda la composición de la «Lógica» que se publicaría en 1843. Entre 1845-47 escribió su obra cumbre «Principios de Economía Política» publicada con gran éxito en 1848 que dominó el ámbito académico de la economía inglesa durante cuarenta años hasta la publicación de la obra de Jevons. En 1859 publicó «La libertad» un tratado sobre la libertad de pensamiento y de costumbres. En 1861 publica «Consideraciones sobre el gobierno representativo» sobre la democracia y la participación de las minorías en el parlamento. En 1865 fue elegido parlamentario por el partido liberal, diputado izquierdista y radical defendió el derecho al voto de la mujer, atacó la esclavitud y apoyó a los sindicatos. En 1869 pu- 
blicó «La sujeción de las mujeres» y en el 1873 salió a la luz su «Autobiografía», año en el que muere en Avignon.

\section{Su obra de juventud: «Ensayos sobre algunas cuestiones dis- putadas en Economía Política»}

Hacia 1830, con poco más de 20 años, J. S. Mill redactó los cinco ensayos que componen su obra titulada «Ensayos sobre algunas cuestiones disputadas en Economía Política», publicada en 1844.

El primero de estos ensayos, «De las leyes del intercambio entre naciones y la distribución de las ganancias del comercio entre países del mundo comercial» es el más famoso, y presenta la teoría de la demanda reciproca en el comercio internacional que supuso un avance de gran importancia respecto al pensamiento de Adam Smith y David Ricardo, al establecer las condiciones para la determinación de los términos del intercambio. J.S.Mill demostró que el reparto de los beneficios del comercio exterior, esto es, la relación real de intercambio, depende de la demanda de cada país de los productos del otro, la llamada demanda recíproca. Mill rechaza lo aranceles proteccionistas, sólo acepta los fiscales o recaudatorios, sin embargo admite que no se bajen los aranceles si no los bajan también los demás países.

El segundo ensayo, «De la influencia del comercio sobre la producción» de importancia comparable al primero, refuta la ley de Say que sostiene que «la oferta crea su propia demanda y la sobreproducción general es imposible». J.S.Mill advirtió que en una economía monetaria en la que existe el dinero que puede ser demandado como activo en sí mismo puede no cumplirse la ley de Say y puede existir sobreproducción. Mill Demuestra el papel del dinero en el ajuste de los mercados.

En el tercer ensayo «Sobre las palabras productivo e improductivo" se ocupó de establecer la distinción entre actividades productivas y no productivas, preocupación presente en la ciencia económica desde la escuela de los fisiócratas. J.S.Mill extiende la distinción no sólo al trabajo sino también al capital y al gasto. En su opinión, el trabajo, el gasto o la producción cuyo ob- 
jeto inmediato es la satisfacción inmediata son improductivos. La idea es que la producción de capital en sentido amplio o de «fuente de disfrute» es el criterio de productividad.

El cuarto ensayo «Sobre los beneficios y el interés» vuelve sobre el esquema ricardiano de distribución de la renta y la idea del «coste de producción» de los salarios. Mill expone la idea de que los beneficios pueden ser incrementados, bien rebajando los salarios o bien reduciendo su coste de producción, es decir, el valor de la fuerza de trabajo o el valor de los bienes que consumen los trabajadores. Esta idea llamativa es parecida a la discusión sobre el plusvalor absoluto y relativo de Marx.

En el quinto ensayo «Sobre la definición de la Economía política y sobre el método de investigación más adecuado para la misma» aborda el estudio de los problemas metodológicos. Su definición más elaborada sobre las leyes de la economía era la siguiente: «las leyes de los fenómenos sociales que derivan de las operaciones combinadas de los seres humanos para la producción de riqueza, en la medida que esos fenómenos no resulten modificados por la persecución de ningún otro objetivo».Mill defiende el método deductivo, abstracto, a priori, para descubrir las leyes de la economía en la línea de lo que más tarde Marshall acuñaría como la cláusula ceteris paribus para la economía neoclásica. Mill hace una defensa de la teoría pero advierte del riesgo de incorporar los modelos teóricos muy simplificados a la política económica que debe aplicarse al complejo mundo real. Cree que la economía es mucho más de lo que puede ser abarcado por una teoría puramente deductiva y así se pondrá de manifiesto en su obra de los «Principios» cuyo título completo es «Principios de Economía Política, con alguna de sus aplicaciones a la filosofía social».

\section{Su obra de madurez: «Principios de Economía política»}

Los «Principios» de J. S. Mill es una obra que relaciona las leyes teóricas con su aplicación práctica. Su objetivo es investigar sobre la naturaleza de la riqueza y las leyes de su producción y distribución así como las causas por las que la sociedad prospera o decae respecto a ese objetivo universal. 
Se compone de cinco libros: «La producción,» «La distribución», «El cambio», «La influencia del progreso de la sociedad sobre la producción y la distribución» y el último libro «Sobre la influencia del gobierno".

En el primer libro J. S. Mill distingue entre factores productivos: Trabajo, capital y materias primas y los requisitos de la producción como es el trabajo tanto corporal como mental y los objetos naturales que pueden ser limitados o ilimitados. Realiza varias taxonomías sobre el trabajo. Una primera refiriéndose al trabajo directo, al trabajo indirecto y al trabajo intelectual; el trabajo indirecto sería aquel destinado a producir materiales que luego emplearán la industria, herramientas o utensilios, aquellas actividades destinadas a la protección de la industria como la construcción de edificios, muelles, graneros, aquellas otras tareas que permiten una mayor accesibilidad al producto, como es el transporte; en esta categoría de trabajo indirecto incluía también la crianza de la población infantil.Una segunda clasificación sería en trabajo productivo e improductivo a la que ya se refirió en su obra de los «Ensayos». La aportación de J.S.Mill está entorno a la clasificación que hace sobre las utilidades producidas por el trabajo. Distingue entre utilidades o propiedades aplicadas a objetos externos que los hacen más aptos para el consumidor, utilidades o propiedades aplicadas a seres humanos que los hacen más útiles a ellos mismos y a los demás, por ejemplo la actividad de los médicos o la educación.Y por último las utilidades no fijadas que sólo consisten en prestar un servicio inmediato, conceder placer o evitar dolor, como por ejemplo el trabajo de un músico o un actor. Estas no pueden considerarse como riqueza generada. Sólo el trabajo productivo produce utilidades incorporadas a los objetos materiales y esto es lo que produce riqueza y acumulación en una sociedad.

Para J. S. Mill el capital es sinónimo de riqueza y es el resultado del ahorro, «el ahorro enriquece a la comunidad al mismo tiempo que a los individuos, mientras que el gasto los empobrece;lo que equivale a decir que la sociedad en general es más rica por todo aquello que gasta en mantener y ayudar al trabajo productivo, pero más pobre por lo que consume en sus placeres». Distingue entre capital fijo y capital circulante que se destruye como tal; todo aumento del capital fijo a expensas del circulante perjudica los intereses de los trabajadores. Habla así 
mismo de las causas de la productividad de los agentes productivos, entre ellas las ventajas naturales, la habilidad y los conocimientos y la seguridad en el trabajo.

Mientras A. Smith hablaba de la división del trabajo, J.S.Mill se refería a la separación de ocupaciones y a distintas formas de combinar el trabajo dando lugar a la civilización industrial limitada por la extensión del mercado. En su opinión la producción es más eficaz si se produce a gran escala y enumera las ventajas del principio de participación por acciones de las Sociedades Anónimas.

En su segundo libro expone como la distribución de la riqueza depende de las instituciones humanas. Realiza un acercamiento a posiciones cercanas al comunismo y socialismo. Según J. S. Mill la filosofía comunista se basa en la propiedad indivisa de los factores de producción y tanto el producto como el trabajo se reparten con igualdad. Dentro del socialismo destaca dos corrientes en su tiempo, el Fourierismo, movimiento que no abole la propiedad privada ni la herencia, admite como elemento de distribución de los productos tanto el capital como el trabajo; primero se asigna una cantidad de subsistencia a cada miembro de la comunidad trabaje o no, luego se reparte el resto entre capital trabajo y talento. El Saintsimonismo defendía que los productos se repartían con desigualdad y cada individuo debe realizar el trabajo para el que esté más capacitado.J.S.Mill estuvo más próximo al pensamiento de Saint-Simón que de Fourier.

Bajo el dominio de la propiedad privada la distribución de los productos depende de dos instituciones, que son: la costumbre y la competencia.

Para J. S. Mill los salarios se determinan por la competencia, esto es por el juego de oferta y demanda en el mercado de trabajo o por la costumbre, como es el caso de la retribución de médicos o abogados. Los salarios deben ser variables, debía fijarse un salario mínimo y un sistema de subsidios que durante 30 o 40 años estuvo dominado por las parroquias.Se preocupa de medidas que hagan desaparecer la pobreza y señala para ello la necesidad de la educación de los hijos de la clase trabajadora. No olvidó referirse a la desigualdad de los salarios de las mujeres.

J. S. Mill desarrollo una teoría sobre los salarios que denominó como «Fondo de salarios» y de la que finalmente se retractó. Según esta teoría los salarios pueden aumentar por pre- 
sión de los sindicatos, no tienen por que ser una cuantía fija, en detrimento de los beneficios, esto puede reducir el crecimiento y llevar a la economía a un estado estacionario pero no catastrófico, la conclusión de esta teoría vendría ser la necesidad de reducir el número de hijos por trabajador.

En cuanto a las ganancias del capital defendía que la causa de esa ganancia es que el trabajo produce más de los precios para su subsistencia y esto depende de dos factores: de la cantidad de trabajo y su productividad y por otro lado de la proporción entre la remuneración del trabajador con la cantidad de producto que produce. La parte de ganancia que es el interés, entendido como la remuneración a la abstinencia es igual en todos los empleos del capital, pero pueden existir desigualdades que vienen dadas por el riesgo de la actividad concreta o por la existencia de monopolios naturales.

En su tercer libro, titulado «El Cambio», J.S.Mill habla del concepto de valor distinguiendo entre valor en uso medido por la utilidad de los bienes y el valor en cambio medido por la capacidad de compra. El valor mide el costo de producción y la ganancia ordinaria y lo que cuesta producir una cosa es el valor del trabajo empleado, aproximadamente la suma de los salarios, la ganancia y los impuestos.

El dinero es la medida de las posesiones y el medio de distribución de los ingresos, el valor del dinero es inverso al movimiento de los precios, introduce el concepto de velocidad de circulación del dinero que depende de la cantidad de dinero en circulación distinto de la cantidad de dinero que se gasta. El dinero entra a un país de dos formas: bien se importa en barras de metal como cualquier otra mercancía, bien se importa como medio de cambio en pago de deudas por productos exportados u otras causas. Habla del crédito como sustitutivo del dinero y de los efectos que este tiene sobre la producción y sobre los precios por cuanto estimula las transacciones. Así como del papel de cheques y de las letras de cambio, éstas, evitan los gastos y los riesgos de transporte de metales preciosos (oro y plata, materiales de los que estaban fabricadas las monedas) de un lugar a otro. Los billetes de banco debían ser emitidos por el gobierno puesto que debía existir una garantía de solvencia de los emisores y por otra parte debían ser los perceptores del señoriaje, es decir, de la ganancia asociada a esa emisión de dinero fidu- 
ciario. El tipo de interés que resulta de la oferta y la demanda de préstamos, determina el valor y el precio de todos los bienes que se compran por la renta que producen, como acciones 0 fondos públicos, la relación entre el interés y el precio de estos títulos siempre son inversos.

El cuarto libro «Influencia del progreso de la sociedad sobre la producción y la distribución». Varias son las causas que esgrime J.S.Mill como desencadenantes del progreso de la riqueza; entre ellas, el aumento gradual de la producción y la población, un crecimiento perpetuado, el aumento de la seguridad de las personas y de la propiedad que propician el aumento de la producción y de la acumulación, el talento comercial, el crecimiento del principio de cooperación, el progreso industrial que hace disminuir el coste de producción y las fluctuaciones de los precios. Para Mill el crecimiento estacionario es una meta que se mueve hay que luchar por avanzar aumentando la producción teniendo en el punto de mira como objetivos mejorar la distribución y alcanzar una remuneración amplia del trabajo, concediendo en este proceso una importancia creciente de la clase obrera.

Su quinto libro «Sobre la influencia del gobierno" trata sobre las funciones del gobierno distinguiendo entre necesarias y facultativas. Las necesarias son inseparables del concepto y son las más habituales, están creadas por ley y tienen un carácter igualitario. Las facultativas están creadas por los gobiernos tienen un carácter discriminatorio o son cuando menos discutibles. Entre las primeras estarían la defensa de la propiedad y el cumplimiento de los contratos que pretenden fijar el marco institucional, y establecer leyes contra el fraude, y por otra parte la represión de la violencia, así podemos hablar de tres funciones fundamentadas en la defensa la seguridad y la justicia.Entre las segundas tendríamos la regulación de las condiciones de los préstamos, los monopolios y las leyes contra las uniones de los obreros para elevar sus salarios.

En este libro J.S.Mill establece los principios generales del sistema impositivo fundamentados en la idea de igualdad. Realizando una clasificación entre los diversos impuestos que él divide en impuestos directos aquellos que recaen sobre el ingreso y los impuestos indirectos o impuestos de consumo. El impuesto sobre la renta que recae sobre el terrateniente, el impuesto sobre la ganancia que recae sobre los capitalistas, el 
impuesto sobre los salarios que reduce el nivel de vida de la clase trabajadora o recae sobre las ganancias cargando al capitalista con un impuesto indirecto.Para J.S.Mill los impuestos más relevantes para la economía son: los impuestos sobre las mercancías puesto que elevan su precio y reducen la calidad de vida del trabajador; los impuestos sobre el sector exterior y los impuestos sobre los sistemas fiscales, esto es, sobre los contratos, fundamentalmente los de compra-venta; sobre las comunicaciones, servicios postales, periódicos; sobre las operaciones de los tribunales; y los impuestos locales destinados a la financiación del gasto público como el alumbrado, la limpieza, etc.

La actuación del gobierno, siempre que esta no fuera adecuada, tendría, sin duda efectos económicos; en el caso de imperfecciones en la protección de la propiedad y de los individuos se generaría inseguridad o bien si se dieran abusos en la presión fiscal o arbitrariedad de los funcionarios públicos en el desempeño de sus tareas esto conllevaría desaliento en la actividad productiva y en la prosperidad económica.

J. S. Mill ponía límites a la actuación del gobierno en la parcela íntima del individuo, en su libertad política y en la división del trabajo, no obstante establecía claramente excepciones al laissez-faire en temas como la defensa de la infancia, la educación, la indigencia, la oferta de servicios para la comunidad, la disminución de las horas de trabajo etc.

\section{Pensamiento clásico en España}

El desarrollo de la Economía política Clásica en España tuvo lugar en el periodo comprendido entre las últimas décadas del siglo XVIII, después de la publicación de la obra de A.Smith, "La riqueza de las naciones» en 1776 y la Revolución francesa y un final también difuso entre la crisis del 98 y la Primera Guerra Mundial.

Almenar, S. (2000) considera cuatro etapas fundamentales en la evolución del pensamiento económico clásico en España: La primera se corresponde con el periodo final de la llustración, la segunda abarca el periodo comprendido entre la Revolución de 1808 y los años cuarenta, la tercera llega hasta el inicio de la Restauración y la cuarta iría desde 1874 hasta 1904/1914. 
En la primera etapa se produce en España un florecimiento intelectual y dentro de la última generación de ilustrados españoles, encontramos un grupo más receptivo hacia las materias económicas, entre ellos Jovellanos, Francisco Cabarrus, Valentín de Foronda.No tenían tampoco una especial formación reglada en economía, sino un interés por el estudio de la literatura económica española y algunas obras extranjeras.

Durante la segunda etapa se intentó llevar a la práctica una nueva organización social liberal en sustitución de la sociedad estamental del Antiguo Régimen, coincidiendo aproximadamente con la consolidación de la Economía política clásica. Parece vislumbrar la existencia de una cierta especialización de algunos autores en temas económicos, tratándolos tanto en el plano teórico como en el aplicado, sobre todo a partir de los años 30, como es el caso de Ramón Cañedo, José Canga Argüelles, Alvaro Florez Estrada entre otros. Esta especialización no fue, sin embargo, consecuencia de una educación reglada o de una profesionalización, la mayor parte de estos autores tuvo una formación universitaria en leyes o en filosofía. Sólo algunos (Canga, por ejemplo) asistieron a clases de economía en la importante cátedra de la Sociedad Económica Aragonesa. La difusión del pensamiento económico en la época se vio condicionada por la censura eclesiástica y gubernativa, así como por los exilios y destierros. Puede decirse que no hubo publicaciones periódicas monográficamente dedicadas a la Economía política hasta los años cuarenta.

La tercera etapa arrancaría precisamente en esos años cuarenta, y se consolidó con el impacto revolucionario de 1848.A lo largo de los treinta años siguientes, la Economía política se convirtió definitivamente en una materia de enseñanza universitaria, pero no en una profesión. Esta nueva etapa se caracteriza por una intensa atención hacia lo foráneo. Lluch y Almenar estudian las corrientes de difusión de las ideas económicas exteriores en España y señalan tres grandes transformaciones acontecidas durante los años cuarenta en los flujos internacionales del pensamiento económico. La primera y más importante es la pérdida de hegemonía de las ideas de Say, la segunda es el fuerte proceso de difusión y aceptación de las obras de Bastiat en España. Paralelamente se observa la fabulosa recepción de las ideas de la Escuela Economista francesa en general. El vacío 
más importante en este periodo es la escasa presencia de los escritos y las ideas de J. S. Mill que no empezaría a utilizarse hasta la Restauración.

La cuarta etapa (1875-1904/1914) comprende el agotamiento doctrinal y personal de la Escuela Economista en España con la introducción de otras perspectivas doctrinales como las corrientes historicistas y reformistas que reubicaron el papel del Estado y las instituciones en el desarrollo económico general y en las relaciones sociales. La desaparición de J.S.Mill en 1873 y J.E.Cairnes en 1875 coincidió con la publicación de las obras de Jevons, Menger, Walras exponentes de la nueva economía. Sin embargo la denominada revolución marginalista tuvo escasísima incidencia en los economistas españoles. Una de las características más destacables del desarrollo del pensamiento económico contemporáneo en España es el retraso con que se incorporó la nueva economía a la enseñanza y la investigación. Esta incorporación se produjo después de la guerra civil, con la puesta en funcionamiento de la Facultad de Ciencias Políticas y Económicas de Madrid en 1943 y la renovación de las enseñanzas en las escuelas de ingenieros industriales y agrónomos.

A lo largo de esta etapa se distinguen cuatro grupos doctrinales. El primero enrraizado con la Escuela optimista mantuvieron una posición deductivista interesados abiertamente por la economía matemática (Aller, Figuerola, Rodriguez, Echegaray). El segundo grupo encarnan una matizada continuidad con la Escuela Economista, con una mayor aceptación de las ideas de J. S. Mill y con una primera recepción historicista (Madrazo, Salvá, Olózaga). El tercer grupo formado por algunos historicistas explícitos (Piernas Hurtado, Alvarez Buylla). Por último un cuarto grupo cercano al cristianismo social.

Aunque en esta época la hegemonía de la Escuela Economista comenzaba a declinar puede interpretarse como un factor del estancamiento del pensamiento económico en España. La mayor parte de los economistas españoles del último tercio del siglo XIX tuvieron un conocimiento superficial de la economía marginalista y neoclásica. Las razones que se aducen son las siguientes: En primer lugar la formación fundamentalmente jurídica o filosófica de los economistas españoles que no incluía una instrucción matemática suficiente. En segundo lugar, los flujos de entrada de las corrientes de pensamiento ex- 
terior estaban muy limitados a la cultura económica francesa o la literatura histórica italiana a través de los cuales recibían información sobre el Historicismo alemán lo que afectó a la difusión de la teoría económica marginalista. En tercer lugar, las tareas docentes de los economistas españoles eran compartidas por la política o el ejercicio profesional como abogados y la investigación cuando existía quedaba en tercer plano. Por tanto, en España se dificultó el proceso de difusión internacional del pensamiento económico, ya que no existían las condiciones más favorables para la recepción inmediata de la nueva econo mía. Así, la Economía política clásica prolongó en España su vigencia decreciente hasta bien entrado el siglo Xx.

\section{Referencias bibliográficas}

BARBE, LL. (1996). El curso de la economía. Grandes escuelas, autores y temas del discurso económico. Madrid: E. Ariel.

LLUCH, E. y ALMENAR, S. (2000). «Difusión e influencia de los economistas clásicos en España (1776-1870)» en FUENTES QUINTANA, E. (dir.): Economía y economistas españoles, vol. 4. Ed. Barcelona: Galaxia Gutenberg,

MILL, J. S. (1844). Ensayos sobre algunas cuestiones disputa das en Economía política. Madrid, 1997: Alianza Editorial.

MILL, J. S. (1848). Principios de Economía Política. México, 1978: Fondo de Cultura Económica.

MILL, J. S. (1873). Autobiografía. Madrid (1986) : Alianza Editorial.

O’BRIEN, D. P. (1989). «Los criterios clásicos de política económica» en Los economistas clásicos. Madrid:Alianza Editorial. SÁNCHEZ HORMIGO, A. (1999). «Estudios preliminares», en J. A. COVERT-SRPING, Escritos saint-simoniacos, I.E.F.

SCHUMPETER, J. A. (1994). Historia del Análisis Económico. Madrid: Ariel.

SCHWART, P. (1968). La «nueva Economía política» de John Stuart Mill. Madrid : Editorial Tecnos. 